\title{
Modelling and Analysis on Noisy Financial Time Series
}

\author{
Jinsong Leng
}

Bradford Street, Mount Lawley, Perth, Australia.

Email: j.leng@ecu.edu.au

Received November 2013

\begin{abstract}
Building the prediction model(s) from the historical time series has attracted many researchers in last few decades. For example, the traders of hedge funds and experts in agriculture are demanding the precise models to make the prediction of the possible trends and cycles. Even though many statistical or machine learning (ML) models have been proposed, however, there are no universal solutions available to resolve such particular problem. In this paper, the powerful forward-backward non-linear filter and wavelet-based denoising method are introduced to remove the high level of noise embedded in financial time series. With the filtered time series, the statistical model known as autoregression is utilized to model the historical times aeries and make the prediction. The proposed models and approaches have been evaluated using the sample time series, and the experimental results have proved that the proposed approaches are able to make the precise prediction very efficiently and effectively.
\end{abstract}

\section{KEYWORDS}

Financial Time Series; Filtering and Denoising; Autoregression; Modelling and Prediction

\section{Introduction}

In last few decades, the analysis of time series has attracted much attention from statistical and machine learning perspectives [1,2], with a variety of applications in different fields $[3,4]$. For example, the traders of hedge funds and experts in agriculture are demanding the precise models to make the prediction of the possible trends and cycles. Even though a number of techniques and models are proposed for analyzing financial time series, however, there are no universal solutions to such specific application, due to its inherent randomness in nature. Also it is very difficult to determine which approach or model is superior to others, since many statistical and machine learning approaches are application-oriented methods.

Many real applications, such as the electric signals and financial time series, consist of high level of white noise and colored noise, making it almost impossible to build the appropriate model(s) for prediction and forecasting. Most of statistical models and machine learning techniques have no capability of noise resistance. Separating the deterministic time series from the noisy raw signal(s) is becoming another obstacle to build the effective prediction models.

To address the problems discussed above, several dif- ferent approaches are proposed in this work. In doing so, two diversified denoising techniques are introduced and evaluated through the comparative studies. Also the autoregression (AR) model is utilized for modeling and prediction.

The major task of this work is to develop the statistical or machine learning (ML) models for analyzing the historical financial time series. Such models should be able to derive the useful knowledge such as patterns and regularities, trends and cycles, so as to make the precise prediction. The major contributions of this paper are summarized as follows:

- Propose two efficient and effective denoising techniques to remove the noise embedded in the time series i.e., non-line low-pass forward-backward filter and wavelet orthogonal projection denoising method.

- Build the AR model with the relative low order, and the excellent performance has been obtained in the experimental analysis.

- Utilize two new criteria-approximate entropy and student-t test to assess the performance of filters and AR model.

The paper is organized as follows: The forwardbackward filter and wavelet denoising method are detailed in Section 2. In Section 3, the AR model is discussed and the performance criterion is described. In 
Section 4, the experimental results are specified and evaluated with the sample financial time series available in book (Analysis of Financial Time Series) [3]. Section 5 concludes the paper.

\section{Filters}

Normally, the financial time series is embedded with high level of noise (random trading behaviors), such as white noise and colored noise. It is very difficult to determine the level of such unknown noise and find the appropriate filtering techniques that can separate the deterministic time series and random events. If the raw noisy time series is less denoised, the prediction model performs poorly due to the high level noise; if the noisy time series is over denoised, the filtered time series loses some genuine features of raw time series. The conventional time series cannot filter such high level noise such as financial time series. Indeed, the effective filtering is dependent on the several factors, e.g., the ability to remove the noise, the types of noise, and the thresholds estimation, etc.

In this paper, two different types of filtering techniques are utilized in this paper: one is the traditional non-linear low-pass filter with forward and backward filtering (FBF) processes; another is the wavelet based denoising method (WLD) for which the time series is projected into orthogonal basis. Also, the measure criterion known as approximate entropy (ApEn) is considered to evaluate the performance of proposed filters.

\subsection{Forward and Backward Filter}

The forward-backward filter (FBF) actually is a matrix with no-linear processing networks. It utilizes the second-order matrix SOS and the scale vector G, by conducting the forward and reverse the filtering processes [5]. The scale $\mathrm{G}$ defines the weights of input samples. The SOS and G are defined by:

$$
\begin{gathered}
S O S=\left(\begin{array}{c}
b_{01} b_{11} b_{21} a_{01} a_{11} a_{21} \\
b_{02} b_{12} b_{22} a_{02} a_{12} a_{22} \\
\cdots \\
b_{0 L} b_{1 L} b_{2 L} a_{0 L} a_{1 L} a_{2 L}
\end{array}\right) \\
G=\left[w_{1} w_{2} w_{3} w_{4} w_{5} w_{6}\right]
\end{gathered}
$$

FBF filters the time series $X$ with the SOS filter described by the matrix SOS and the vector G. After filtering in the forward direction, the filtered sequence is then reversed and run back through the filter. In this project, the Butterworth second-order filtering is used for filtering the time series.

The example of FBF filtering process with the financial time series is illustrated in Figure 1.

\subsection{Wavelet-Based Denosing}

Wavelet theory is an emerging new signal processing technique in recent two decades [6,7], which is called the mathematical microscope due to it high recognition accuracy in both time domain and frequency spectrum. With the scaling factor $a$ (dilation factor) and translation parameter $b, a, b \in \mathrm{R}$, and $a \neq 0$. The prototype wavelet is scaled and translated. The wavelet function can be expressed as:

$$
\Psi(t) a, b=\frac{1}{\sqrt{a}} \Psi\left(\frac{t-b}{a}\right)
$$

$1 / \sqrt{a}$ is the normalized factor, so as to make sure for all a, b, $\Psi(t)$ has the unit energy.

The concept of multiresolution was proposed by Mallat and Meyer in 1989 [8], meaning that one signal can be decomposed into the orthogonal projections and can also be fully reconstructed. The components of the decomposition are divided into the approximation (a) and details (d) at different levels. The approximation represents the major feature of the signal and the details describe the detailed changes and noise. The time series can be denoised by removing some ingredients from the projections in details.

The example of wavelet denoising is given in Figures 2 and 3.

\subsection{Performance Measurement}

To evaluate twofilters proposed above, two measur ements are introduced: 1). One indicator is thet rate of autoregression model (AR), the details about AR model are available in Chapter 3. 2). Another criterion known as the approximate entropy (ApEn) [9] is also introduced.

The major ability of ApEn is to evaluate the time series by quantifying the amount of regularity and the unpredictability of fluctuations. The successful applications have been found in EEG signal diagnosis [10] and in financial time series [11]. In [11], it was reported that the uncertainty events such as the Asiarfinancial crisis can be detected by analyzing Hang Seng index. There are two parameter $m$ and $r$ in ApEn. The value of $m$ is between $2-3$, and the value of $r$ is about $0.2 \nsucc$ ( $\sigma$ is the value of standard deviation of the time series).

The smaller of ApEn, the better regularity and trends of the time series.

Here, an example is given to assess the performance of two filters using AR model and ApEn. Firstly, I compare the quality offiltering with time series L0 using AR(p) model, detailed as below (FPE-final prediction error, MSE—-mean square error):

- For original signal L0, the results with AR (6) are: Fit to estimation data $-0.3754 \%$, FPE -0.0002323 , MSE -0.0002308 . Even AR with order of 30, the results 

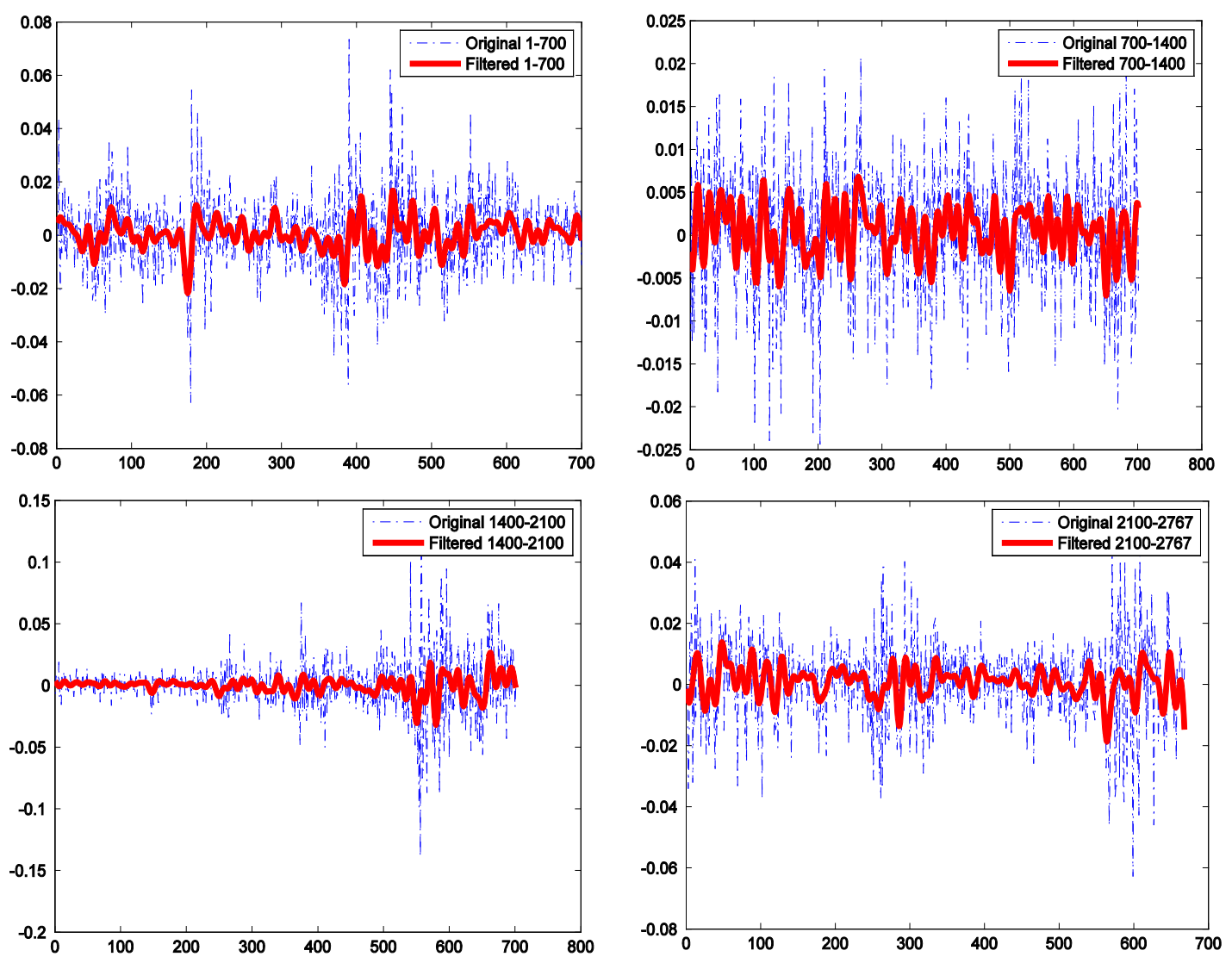

Figure 1. Original and filtered times series (FBF).

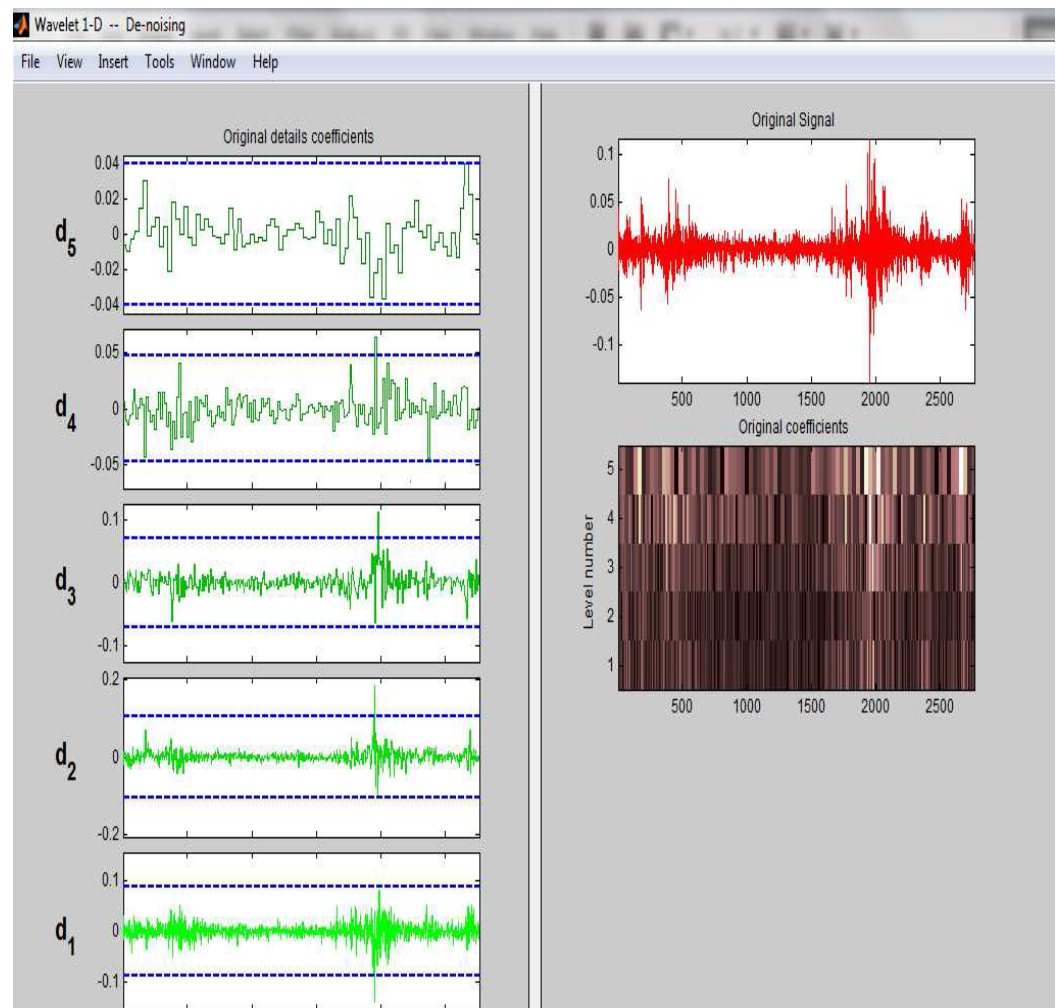

Fgure 2. Approximate and details of times series. 


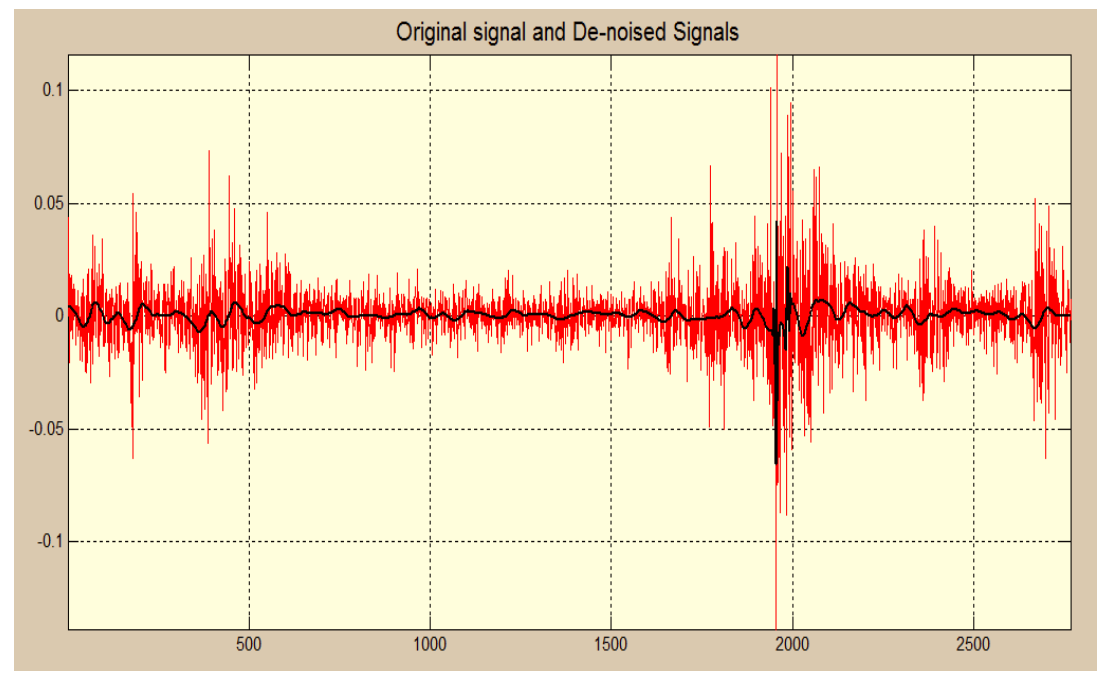

Figure 3. Original and filtered times series (Wavelet).

are still very poor: Fit to estimation data-1.202\%, FPE-0.0002344, MSE-0.000227.

- For the forward-backward filtered L0, the results with AR (6) are: Fit to estimation data-99.98\%, FPE1.557e-12, MSE-1.548e-12.

- For the wavelet-based denoised L0, the results with AR (6) are: Fit to estimation data-99\%, FPE6.884e-10, MSE-6.842e-10.

- It is almost impossible to build the AR model with the original signal (fit rate (r) is too low, $0<\mathrm{r}<22 \%$ ). After filtering using FBF or wavelet, $\operatorname{AR}(\mathrm{p})$ model can be built and used to make the prediction. The performance (almost $100 \%$ fit rate) of AR is excellent using the filtered time series by FBF or wavelet.

Also two filters can be evaluated by another criterion ApEn. As shown in Figure 4, we can find the ApEn value of original signal is significant bigger at $r=0.2$. Figure 5 suggests that wavelet-based denoising is slightly better than that of forward-backward filter. However, AR model indicates that FBF outperforms a little better than wavelet-based denoising method.

Due to space limit, I only use thefiltered datasets by FBF to conduct the model training and prediction.

\section{Model}

\subsection{Autoregression}

Let $\{\mathrm{r} 0, \mathrm{r} 1, \ldots, \mathrm{rt}, \ldots\}$ be a time series, the pth order autoregressive polynomial model AR(p) model [1,2] is defined by:

$$
r(t)=\varnothing_{0}+\varnothing_{1} r_{t-1}+\ldots+\varnothing_{p} r_{t-p}+\varnothing_{1} r_{t-1}+\ldots+\varnothing_{p} r_{t-p}+a_{t}
$$

where $p$ is a non-negative integer and $\emptyset_{\mathrm{p}}$ are the coefficients in the autoregressive model, $\left\{\mathrm{a}_{\mathrm{t}}\right\}$ is assumed to be a white noise series with mean zero and variance $a_{t}{ }^{2}$.

The parameters of the AR(p) can be estimated by sev-

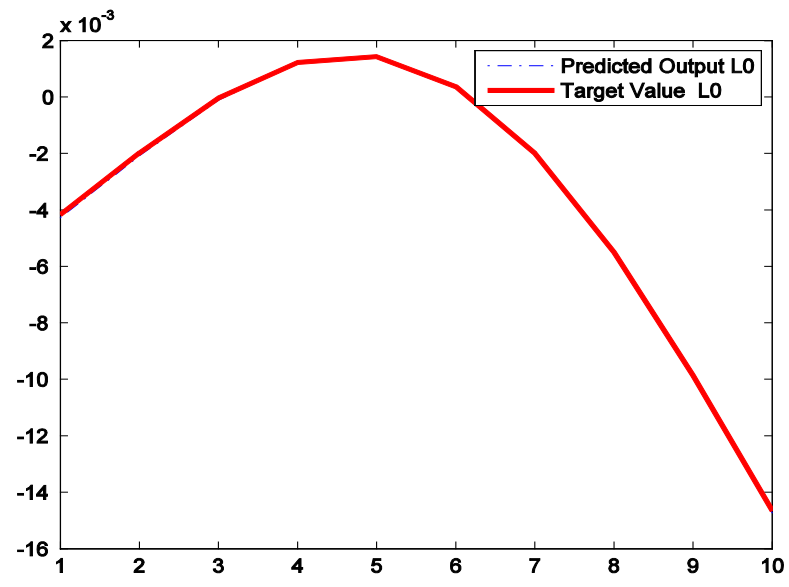

Figure 4. Predicted output and target value (1).

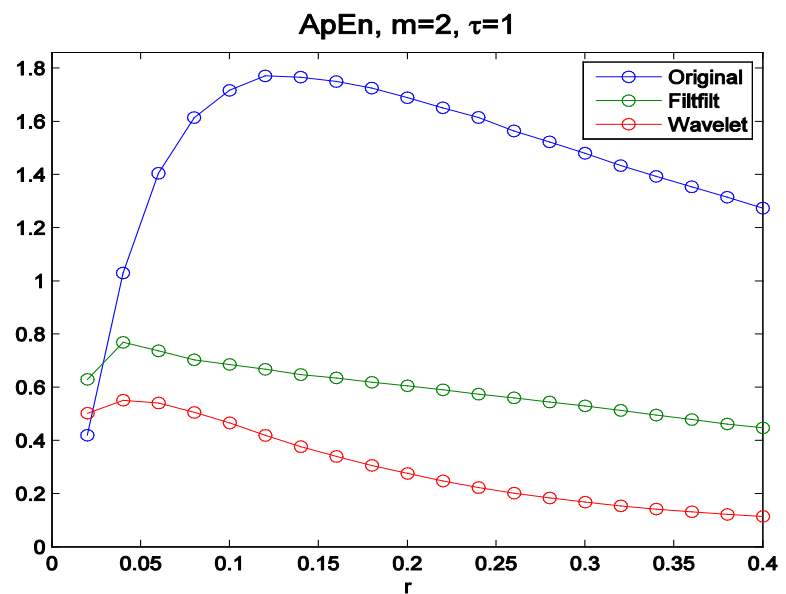

Figure 5. ApEn value of original and filtered signal.

eral ways to replace the theoretical covariance, including the forward-backward approach, the Least Squares method, the Yule-Walker method, etc. In this project, the 
forward-backward approach is used to estimate the parameters of the AR(p) model. The AR(p) can be trained and validated by sample time series. Once the $\operatorname{AR}(\mathrm{p})$ is built, it can be used to predict the future trends and cycles.

\subsection{Student-T Test}

One statistical criterion known as Student-t test is used to quantify the "goodness" of the prediction. This is the statistical criterion to make a test decision for paired time series. The test decision for the null hypothesis is to be made in terms of the acceptance or rejection (h: 0 or 1 ) and the confidence level ( $\mathrm{p}$-value). For example, if $\mathrm{p}=$ 0.95 , the probability of rejecting null hypothesis is only $5 \%$, and thus the prediction will be regarded as the high quality.

\section{Experiments}

There are two parameters in AR to be tuned, i.e., the length of prediction (output), and the order $\mathrm{p}$ in AR. The order of $p$ is required to be tuned in advance. Also it is not easy to evaluate the quality of the AR model, I include a function to calculate the Student-t test values and thus can adjust the order of AR model easily. The order of $\mathrm{p}$ is tuned according to the student-t p-values. After several times trials, I find the best solution to all time series in datasets when the order of $\mathrm{p}$ is 18 . Also I found that AR model can predict 10 - 15 days perfectly, but it is getting worse when the prediction period is more than 20 days. So I define the prediction period is 10 days.

The last 10 records in the time series in dataset 1 are retained for testing, while the rest of the records (total length - testing length) is used for training and validation.The prediction and the filtered testing is a good matching, as shown in Figure 4.

The p-value of this experiment is excellent (0.998), which indicates that the prediction of AR model is precisely matched to testing records.

To further evaluate the proposed methods, additional testing is conducted by making prediction at any point of time series.

For example, the financial time series $(1-2097)$ is used for training and next 10 days (2098-2107) as the testing data, as shown in Figure 6. The related p-values are 0.982 and MSE 1.81e - 08.

Another example is given in Figure 7, the related training data are from 1 to 2157 and the testing data are from 2158 to 2167. From Figures 6 and 7, we can see the predicted data and testing data is matched precisely. The related p-values are 0.915 and MSE 4.03e-09.

\section{Conclusions}

In this paper, several methods have been used to com-

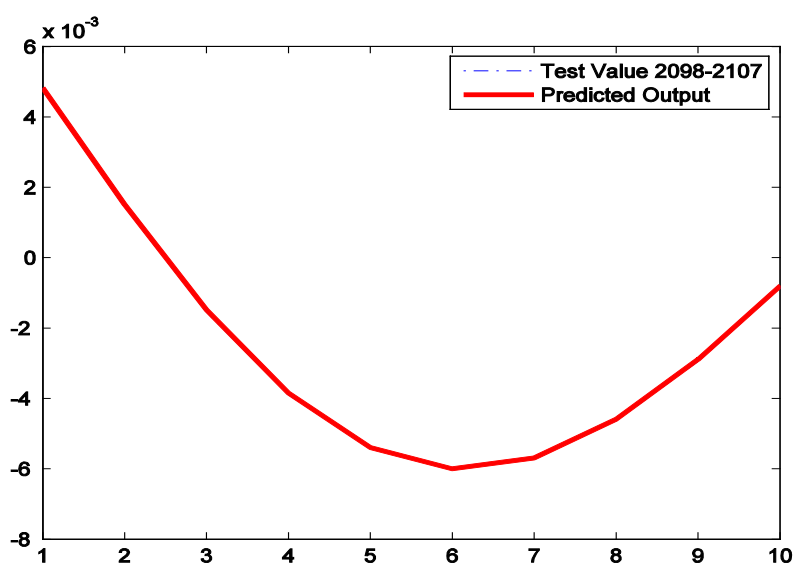

Figure 6. Predicted output and target value (2).

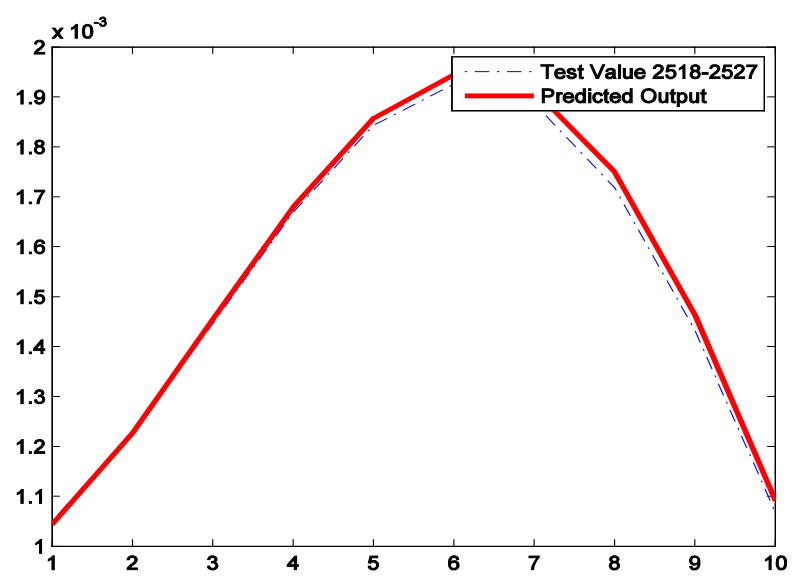

Figure 7. Predicted output and target value (3).

plete the tasks, i.e., two denoising filters, AR prediction models, and two assessment criteria. The approaches of two filters (filtering and wavelet denoising) are proposed from different perspectives.

The advantage of using different approaches and models can provide alternative solutions and the comparative studies to this specified application. The quality measure known as approximate entropy is introduced to assess the quality of preprocessing methods; another statistical criterion known as Student-t test is used for quantifying the prediction models. The software codes for all models and approaches have been developed and tested with two sample datasets under Matlab. The experimental results and related performance are excellent.

In summary, there are some challenges and difficulties in this work, outlined as follows:

- Further analysis is needed to analyze: which filter is better?

- The back-propagation neural networks is to be considered as alternative model, so as to provide alternative

- The use of different portfolio analysis models is 
needed to conduct the analysis folio optimization.

In a word, it is clear that the satisfactory results have been obtained; indicating that the proposed approaches and criteria are very effective in analyzing the big finance time series. If the prediction models can be trained using more systematic samples (different cycles and scenarios), the trained models should be more smart and adaptive.

\section{REFERENCES}

[1] G. U. Yule, “On a Method of Investigating Periodicities in Disturbed Series," Philosophical Transactions of the Royal Society of London, Vol. 226, 1927, pp. 267-298. http://dx.doi.org/10.1098/rsta.1927.0007

[2] T. C. Fu, "A Review on Time Series Data Mining," Engineering Applications of Artificial Intelligence, Vol. 24, 2011, pp. 164-181. http://dx.doi.org/10.1016/j.engappai.2010.09.007

[3] R. S. Tsay, "Analysis of Financial Time Series,” John Wiley \& Sons, Inc., New York, 2010.

[4] D. B. Percival and A. T. Walden, "Wavelet Methods for Time Series Analysis,” Cambridge University Press, Cambridge, 2000. http://dx.doi.org/10.1017/CBO9780511841040

[5] F. Gustafsson, "Determining the Initial States in For-
ward-Backward Filtering," IEEE Transactions on Signal Processing, Vol. 44, No. 4, 1996, pp. 988-992. http://dx.doi.org/10.1109/78.492552

[6] I. Daubechies, “Ten Lectures on Wavelets," Society for Industrial and Applied Mathematics, Philadelphia, 1992. http://dx.doi.org/10.1137/1.9781611970104

[7] C. K. Chui, “An Introduction to Wavelets,” Academic Press, 1992.

[8] S. G. Mallat, "A Theory for Multiresolution Signal Decomposition: The Wavelet Representation,” IEEE Transactions on Pattern Analysis and Machine Intelligence, Vol. 11, 1989, pp. 674-693.

http://dx.doi.org/10.1109/34.192463

[9] S. M. Pincus, "Approximate Entropy as a Measure of System Complexity," National Academy of Sciences of the United States of America, Vol. 88, No. 6, 1991, pp. 2297-2301. http://dx.doi.org/10.1073/pnas.88.6.2297

[10] S. M. Pincus, I. M. Gladstone and R. A. Ehrenkranz, “A Regularity Statistic for Medical Data Analysis,” Journal of Clinical Monitoring and Computing, Vol. 7, No. 4, 1991, pp.335-345.

[11] S. M. Pincus and E. K Kalman, "Irregularity, Volatility, Risk, and Financial Market Time Series," Proceedings of the National Academy of Sciences, Vol. 101, No. 38, 2004, pp. 13709-13714. http://dx.doi.org/10.1073/pnas.0405168101 